\title{
Optical and Electronic Properties of Semiconductor 2D Nanosystems: Self-consistent Tight-binding Calculations
}

\author{
ANDREA REALE, ALDO DI CARLO*, SARA PESCETELLI, MARCO PACIOTTI and PAOLO LUGLI \\ INFM-Dipartimento di Ingegneria Elettronica, Università di Roma "Tor Vergata", 00133 Roma, Italy
}

\begin{abstract}
A tight-binding models which account for band mixing, strain and external applied potentials in a self-consistent fashion has been developed. This allows us to describe electronic and optical properties of nanostructured devices beyond the usual envelope function approximation. This model can be applied to direct and indirect gap semiconductors thus allowing for instance the self-consistent calculation of band profile and carrier control in pseudomorphic InGaAs/GaAs HEMTs and SiGe/Si MODFETs.
\end{abstract}

Keywords: Semiconductors, nanostructures, electronic band structure, tight-binding, optical properties

\section{INTRODUCTION}

Electronic and optical properties of semiconductor nanostructures based on homo- and heterojunctions have been investigated theoretically by means of a variety of tools. These range from $a b$ initio approaches [1], which are very precise but require a large computational effort and consequently, are limited only to very small nanostructures, to approximate but easy-to-handle and fast methods such as for example those based on the envelope function approximation (EFA) [2]. In its simplest form, the EFA leads to the evaluation of the energy levels of nanosystems by simply solving a one-electron Schrödinger equation where each semiconductor is described in terms of effective masses and band edges. Such an approach and its generalizations, have been very successful [2-4], even though several problems still cannot be easily handled within the EFA context. The empirical tight binding method (TB) [5-8] has been shown to be a valid alternative to EFA, since it improves the physical content in the description of the nanostructure with respect to EFA without requiring a much higher computational effort. In particular, it allows us to treat indirect-gap semiconductors, heterostructures made by indirect/direct materials and to describe very thin nanostructures [5-7, 9]. Other advantages of TB with respect to EFA consist in the realistic description of the band structure of the whole Brillouin zone and in the possibility to use

\footnotetext{
* Corresponding author.
} 
different Bloch basis functions for each component material of an heterosystem. TB has been mainly used in the calculation of the electronic properties of nanostructures without taking into account selfconsistent charge redistribution, which is an important requirement when we deal with real systems. However, very recently, we have shown [10] that a self consistent tight-binding procedure can be defined. In this paper we will apply the selfconsistent tight-binding approach to realistic nanostructures such as pseudomorphic HEMT and we will show how the method can be easily extended to handle the case of indirect band gap material such as in SiGe MODFETs.

\section{SELF-CONSISTENT TIGHT BINDING}

In this section we discuss the self-consistent tightbinding model for a system where symmetry is broken in one direction, for example the growth axis $(z)$. The wave function $\left|E, \boldsymbol{k}_{\|}\right\rangle$can be written as linear combination of planar Bloch sums, $|\alpha, m\rangle$ [11]

with

$$
\left|E, \boldsymbol{k}_{\|}\right\rangle=\sum_{\alpha, m} C_{\alpha, m}\left(\boldsymbol{k}_{\|}, E\right)|\alpha, m\rangle
$$

$$
|\alpha, m\rangle=\frac{1}{\sqrt{N}} \sum_{\boldsymbol{R}_{\|}^{m}} e^{i \boldsymbol{k}_{\|} \cdot \boldsymbol{R}_{\alpha \|}^{m}}\left|\alpha, \boldsymbol{R}_{\alpha}\right\rangle,
$$

where $\left|\alpha \boldsymbol{R}_{\alpha}\right\rangle$ is a localized orbital, $\boldsymbol{k}_{\|}$is the in-plane wave vector and $N$ is the number of unit cells in the atomic plane. The subindex $\alpha$ refers both to the basis atom index and to the atomic orbital index. The lattice vector, $\boldsymbol{R}_{\alpha}=\boldsymbol{R}+v_{\alpha}$, (where $\boldsymbol{v}_{\alpha}$ is the basis atom displacement), can be written as $\boldsymbol{R}_{\alpha}=m \boldsymbol{d}+\boldsymbol{R}_{\alpha \|}^{m}$ where $m$ is an integer, $\boldsymbol{d}$ a vector parallel to the growth direction with modulus equal to the distance between two atomic planes and $\boldsymbol{R}_{\alpha \|}^{m}$ is a vector on the $m$-th atomic plane. For a given $\boldsymbol{k} \|$, the eigenstates $E$ are calculated by solving the secular equation $\left(H+V_{H}\right)\left|E, \boldsymbol{k}_{\|}\right\rangle$ $=E\left|E, \boldsymbol{k}_{\|}\right\rangle$where $H$ is the system tight-binding hamiltonian and $V_{H}$ is the Hartree potential. In order to calculate electronic and optical properties for real heterosystems, the presence and possible rearrangement of free charges has to be taken into account. The influence of the electronic charge rearrangement can be included at a Hartree level by solving the Poisson equation for the Hartree potential, $d^{2} V_{H} / d z^{2}=-\rho(z) / \varepsilon$, where $\varepsilon$ is the static dielectric constant. The charge density $\rho(z)$ is defined by the square of the wave function averaged over a layer $z$ and weighted by the Fermi function $f(E)$ :

$$
\rho(z)=\frac{e}{(2 \pi)^{2}} \int d \boldsymbol{k}_{\|} \sum_{n}\left|\Psi_{n, \boldsymbol{k}_{\|}}\right|^{2} f\left(E_{n}\right)
$$

where $e$ is the electron charge and $n$ labels the energy levels for a given $\boldsymbol{k}_{\|}$. A full $\boldsymbol{k}_{\|}$integration is performed in the 2D Brillouin zone by using the special $k$-points technique in the irreducible wedge [12]. The convergence of this integration has been obtained by using 5 special points with $\left|\boldsymbol{k}_{\|}\right| \leq 0.06$ $2 \pi / a$ for direct band gap material. To achieve selfconsistency in indirect band gap material we use 8 points with $\left|\boldsymbol{k}_{\|}\right| \leq 0.22 \pi / a$.

The Poisson and Schrödinger equations in the tight-binding representation are iteratively solved until convergence is reached. An open-chain (infinite well) boundary condition is used for Schrödinger's equation. In order to avoid influences on calculated electronic levels, boundaries are chosen far away from the nanostructure region where the density of conduction electrons or valence holes is high. Although a better choice for the boundary condition is provided by complex band structure states as explained in Ref. [11], for all the situations discussed here the open chain condition represents a valid and simple choice. In order to speed up the self-consistent algorithm we have introduced a hybrid method to diagonalize the tight-binding hamiltonian which uses a standard (LAPACK [13]) routine to calculate eigenvalues and an inverse iteration scheme to calculate eigenvectors. The initial conditions for Schrödinger and Poisson equations are obtained self consistently in the effective mass approximation. 
When optical properties are of interest, one can make use of the Kubo formula to define the susceptibility tensor which is related to the currentcurrent response function of the electromagnetic perturbation $[10,14]$.

\section{RESULTS}

In order to show some results obtained with TB coupled with carrier redistribution, we consider several HEMT-like structures.

If we consider a typical device with a $430 \AA n$ doped $\left(n=10^{18} \mathrm{~cm}^{-3}\right)$ cap layer, $20 \AA$ undoped spacer in $\mathrm{Al}_{0.3} \mathrm{Ga}_{0.7} \mathrm{As}, 100 \AA$ GaAs $2 \mathrm{D}$ channel and $\mathrm{Al}_{0.3} \mathrm{Ga}_{0.7} \mathrm{As}$ buffer, $\mathrm{TB}$ shows small improvements in band profile, charge density and energy levels with respect to EFA. However, a more critical situation is obtained when higher $\mathrm{Al}$ concentrations are considered in the cap layer, which bring the barrier region to be almost indirect gap (e.g., $E_{\Gamma}=E_{X}$ ). Thus, we consider in Figure 1 a HEMT similar to the previous one, but with $\mathrm{Al}_{0.45} \mathrm{Ga}_{0.55} \mathrm{As}$ as cap and spacer layer material. It becomes evident (see Fig. 2) that EFA completely fails in describing the charge redistribution between the $2 \mathrm{D}$ channel and the parasitic channel which forms due to the large $X$ valley contribution to the charge density.

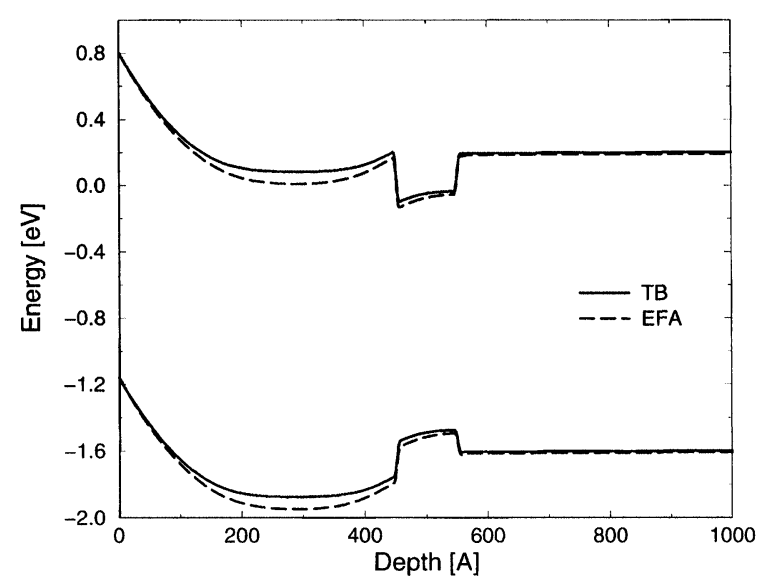

FIGURE 1 Self consistent band profile of a $\mathrm{Al}_{0.45} \mathrm{Ga}_{0.55} \mathrm{As} /$ $\mathrm{GaAs} / \mathrm{Al}_{0.3} \mathrm{Ga}_{0.7} \mathrm{As}$ HEMT structure

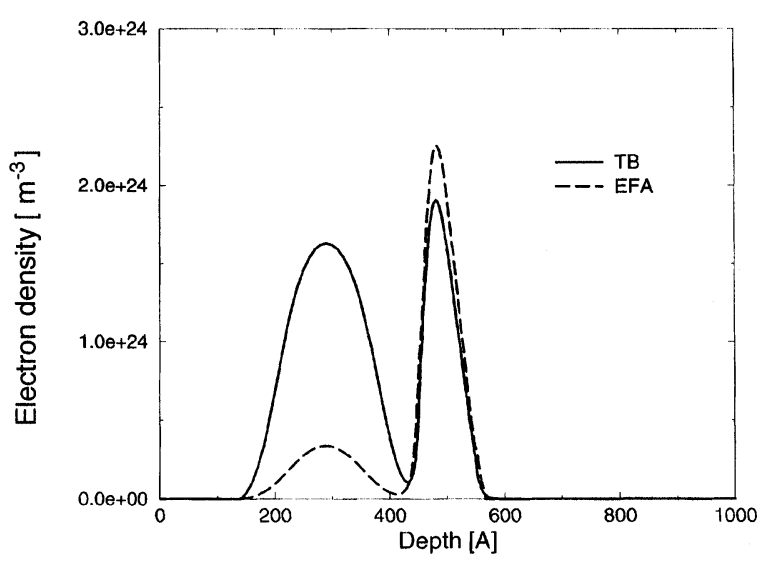

FIGURE 2 Self consistent charge density of the HEMT of Figure 1

The second example refers to a pseudomorphic (PM) structure consisting of a $360 \AA n$-doped $\left(n=10^{18} \mathrm{~cm}^{-3}\right) \quad \mathrm{Al}_{0.2} \mathrm{Ga}_{0.8} \mathrm{As}, 20 \AA$ undoped $\mathrm{Al}_{0.2} \mathrm{Ga}_{0.8} \mathrm{As}$, a $265 \AA$ strained well of $\mathrm{In}_{0.15 \text { - }}$ $\mathrm{Ga}_{0.85} \mathrm{As}$ and a GaAs substrate. Such a modulation-doped structure is typical for PM HEMT's, a device of great importance in the microwave field [15]. In this calculation the background doping charge is simply added to the free charge as given by Eq. (3). The self-consistent potential profile of the structure for two applied gate voltages of -0.6 and $0.0 \mathrm{~V}$ respectively is shown in Figures $3 \mathrm{a}$ and $3 \mathrm{~b}$, respectively. Strain effects in the InGaAs region, which are taken into account by scaling the hamiltonian matrix elements [16], split the $H H$ and $L H$ valence bands. Here the labels $H H$ and $L H$ refer to the character of the valence band in the growth direction. In the parallel direction the uppermost valence band has a light hole character
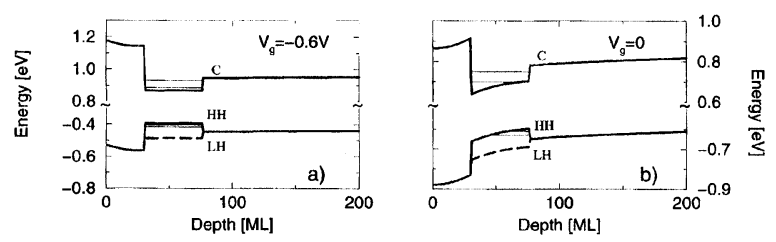

FIGURE 3 Self consistent band edge profile of the $\mathrm{Al}_{0.2-}$ $\mathrm{Ga}_{0.8} \mathrm{As} / \mathrm{In}_{0.15} \mathrm{Ga}_{0.85} \mathrm{As} / \mathrm{GaAs}$ pseudo-morphic structure at applied gate voltages of $-0.6 \mathrm{~V}$ (a) and $0.0 \mathrm{~V}$ (b). The depth is measured in monolayers [ML]. 
while the other a heavy hole one. Strain also induces large changes on the effective masses of the valence bands. For the unstrained situation we have $m_{H H}=0.4475 m_{0}$ and $m_{L H}=0.06481 m_{0}$, while for bulk $\operatorname{In}_{0.15} \mathrm{Ga}_{0.85} \mathrm{As}$ strained on GaAs the effective masses are $m_{H H}[001]=0.4328 m_{0}, m_{L H}$ $[001]=0.09596 m_{0}, m_{L H}[100]=0.08599 m_{0}$ and $m_{H H}[100]=0.1391 m_{0}$. In the confined system, the hole energy levels present a different in-plane mass which depends on the energy. For an applied potential of $-0.6 \mathrm{~V}$ the first three hole levels effective masses are $m_{h 1}[100]=0.10092 m_{0}, m_{h 2}$ $[100]=0.14799 m_{0}, m_{h 3}[100]=0.2421 m_{0}$, respectively. We observe that carrier confinement induces an enhancement of the effective mass compared to strained bulk material, a consequence of band non-parabolicity.

The calculated photoluminescence spectra for several applied potentials are shown in Figure 4. Since the $H H$ quantized levels are now above the $L H$ states, the luminescence transitions occur between conduction levels and the heavy hole levels. For an applied voltage of $-0.6 \mathrm{~V}$, which corresponds to a nearly flat InGaAs band, the main luminescence peaks are related to the $E_{1} \rightarrow H H_{1}$ and $E_{2} \rightarrow H H_{2}$ transitions. This is in agreement with the selection rules $\Delta_{n m}=0$, where $n$ labels the conduction states and $m$ the valence states [2]. The emitted light is mainly

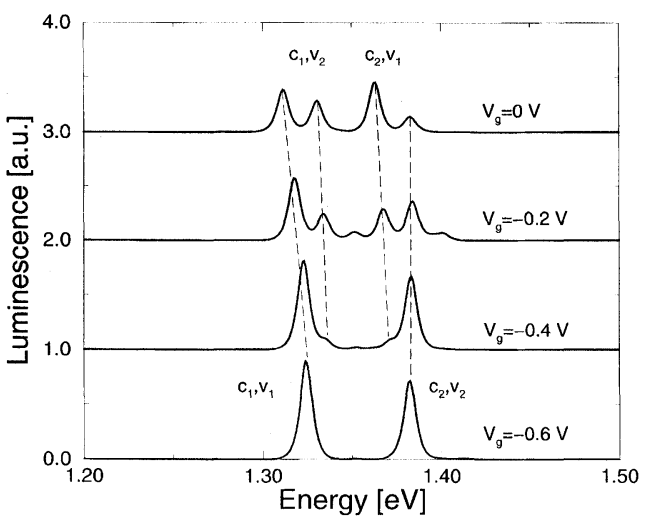

FIGURE 4 Photoluminescence spectra of the pseudomorphic structure for several applied voltages. The dashed lines follow the different luminescence peaks. polarized in the in-plane direction since the levels have essentially a heavy-hole character. When the applied potential is reduced, two other peaks form which correspond to the $E_{1} \rightarrow H H_{2}$ and $E_{2} \rightarrow$ $H H_{1}$ transitions. Indeed, by decreasing the applied potential, the increasing channel electron density induces a sizable band bending which is responsible for the loss of symmetry (see Fig. 3b) and the transitions $E_{1} \rightarrow H H_{2}$ and $E_{2} \rightarrow H H_{1}$ are no longer forbidden. We also notice the presence of a red shift (quantum Stark shift) of the $E_{1} \rightarrow H H_{1}$ transition due to the presence of an electric field as the applied potential reduces from -0.6 to $0.0 \mathrm{~V}$.

As final example we show in Figure 5 the calculated band profile (a) and charge density (b) of a $p$-type $\mathrm{SiGe/Si} \mathrm{MODFET.} \mathrm{Strain} \mathrm{is} \mathrm{respon-}$ sible for the splitting of heavy and light hole bands
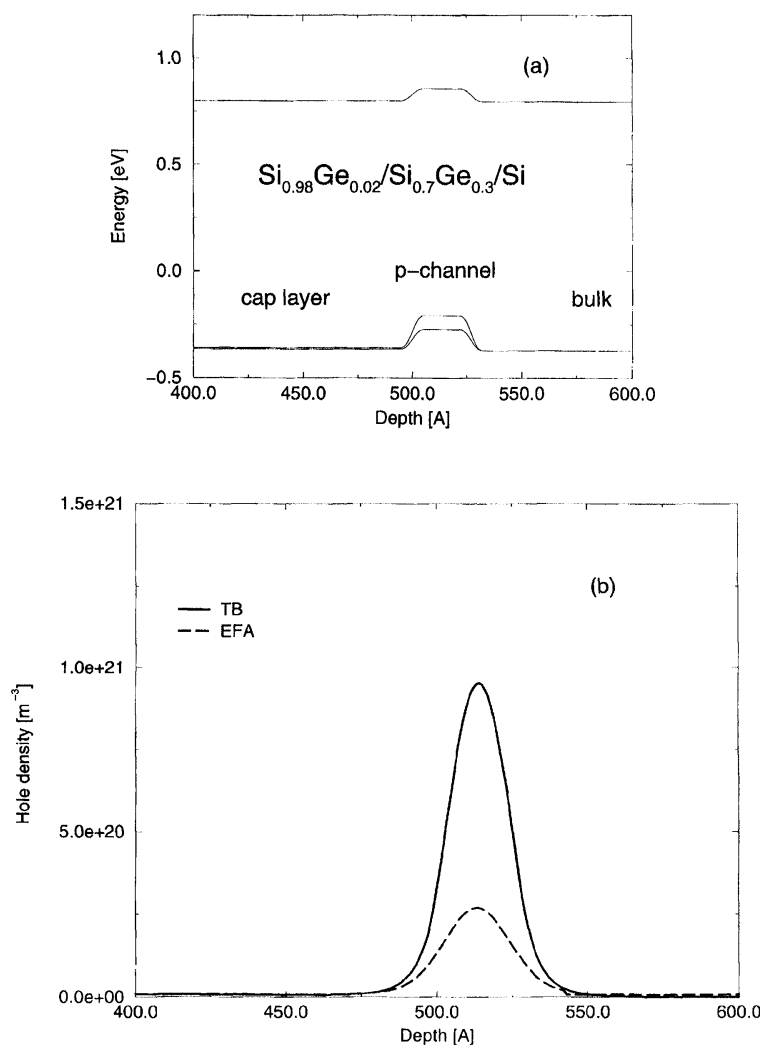

FIGURE 5 Self consistent band profile (a) and charge density (b) of a $\mathrm{Si}_{0.98} \mathrm{Ge}_{0.02} / \mathrm{Si}_{0.7} \mathrm{Ge}_{0.3} / \mathrm{Si} p$-channel MODFET structure. 
as in the previous case. The charge density shows a strong difference between TB and EFA. This is is mainly due to the high non-parabolicity and anisotropy of the valence band.

In conclusion, we have shown that a selfconsistent tight-binding approach can be used to evaluate the electronic structure and optical properties of semiconductor nanostructures. This represents a further step, with respect to the envelope function model, towards an ab initio calculation of such properties.

\section{References}

[1] Wood, D. M., Wei, S.-H. and Zunger, Alex. (1988). Phys. Rev., B37, 1342; Park, C. H. and Chang, K. J. (1993). Phys. Rev., B47, 12709.

[2] Bastard, G. (1988). Wave mechanics applied to semiconductor heterostructure: Les Edition de Physique, Les Ulis Cedex.

[3] White, S. R. and Sham, L. J. (1981). Phys. Rev. Lett., 47, 879; Altarelli, M. (1983). Phys. Rev. B., 28, 842.

[4] Los, J. (1996). Phys. Rev., B53, 4630.

[5] Boykin, T. B., van der Wagt, J. P. A. and Harris, J. S. (1991). Phys. Rev., B43, 4777.

[6] Di Carlo, A. and Lugli, P. (1995). Semicon. Sci. Technol., $10,1673$.

[7] Schulman, J. N. and Chang, Y. C. (1985). Phys. Rev., B31, 2056.

[8] Slater, J. C. and Koster, G. F. (1954). Phys. Rev., 94, 1498; Bullet, D. W. (1980). Solid State Physics, 35, 129; Majewski, J. A. and Vogl, P. (1989). The structure of binary compounds, Amsterdam: Elsevier, 1989. Edited by F. R. de Boer and D. G. Pettifor.

[9] Zunger, A., Yeh, C.-Y., Wang, L.-W. and Zang, S. B. (1994). Proceedings ICPS-22, 1763.

[10] Aldo Di, Carlo, Pescetelli, S., Paciotti, M., Lugli, P. and Graf, M. (1996). Solid State Communications 98, 803.

[11] Di Carlo, A., Vogl, P. and Pötz, W. (1994). Phys. Rev., B50, 8358 .

[12] Froyen, S. (1989). Phys. Rev., B39, 3168.

[13] Anderson, E., Bai, Z., Bischof, C., Demmel, J., Dongarra, J., Du Croz, J., Greenbaum, A., Hammarling, S., McKenney, A., Ostrouchov, S. and Sorensen, D. (1992). LAPACK User's Guide, Philadelphia: SIAM.

[14] Graf, M. and Vogl, P. (1995). Phys. Rev., B51, 4940.

[15] Morkoç H., Unlu, H. and Ji, G. (1991). Principles and Technology of MODFETS, Chichester: John Wiley \& Sons.

[16] Priester, C., Allan, G. and Lannoo, M. (1988). Phys. Rev., B37, 8519 .

\section{Authors' Biographies}

Andrea Reale was born in Italy in 1971 and received the degree in Electronic Engineering from the University of Rome "Tor Vergata" in 1997. He is currently working in the Optoelectronics Laboratory of the Electronic Engineering Departement of the same University, supported by a grant from CSELT-INFM collaboration. His interests include MQW-Semiconductor Optical Amplifiers, optical switches and self consistent band structure characterization of semiconductor devices.

Aldo Di Carlo graduated in Physics at the University of Roma, Italy, in 1991, obtained the Ph.D. degree in Physics in 1995 at the Walter Schottky Institute of the Technical University of Munich (Germany). He is currently researcher at the El. Eng. Dept. of the University of Rome "Tor Vergata", where his work is mainly focused on the theoretical study of optical and transport processes in semiconductor nanostructures, devices and polymers.

Paolo Lugli born in Carpi (Italy) in 1956, obtained his Laurea in Physics at the University of Modena in 1979 and his Ph.D. in El. Eng. at Colorado State University in 1985 . He is currently Full Professor of Optoelectronics at the University of Rome "Tor Vergata". His main field of activity is the theoretical study and numerical simulation of semiconductor nanostructures and devices.

Marco Paciotti born in Rome in 1966, received the degree in Electronic Engineering in 1995 at the University of Rome "Tor Vergata". His main interests concern the modeling and the numerical simulation of semiconductor devices, with a particular knowledge in self consistent calculation of energy structure and charge redistribution. Currently at "Telecom Italia s.p.a.".

Sara Pescetelli born in Rome (1964), graduated in Physics in 1995 at the University of Rome "Tor Vergata". Her main field of activity concerns tight binding calculations of electronic and optical properties of semiconductors. Currently she is working into the El. Eng. Dept. of the University of Rome "Tor Vergata". 

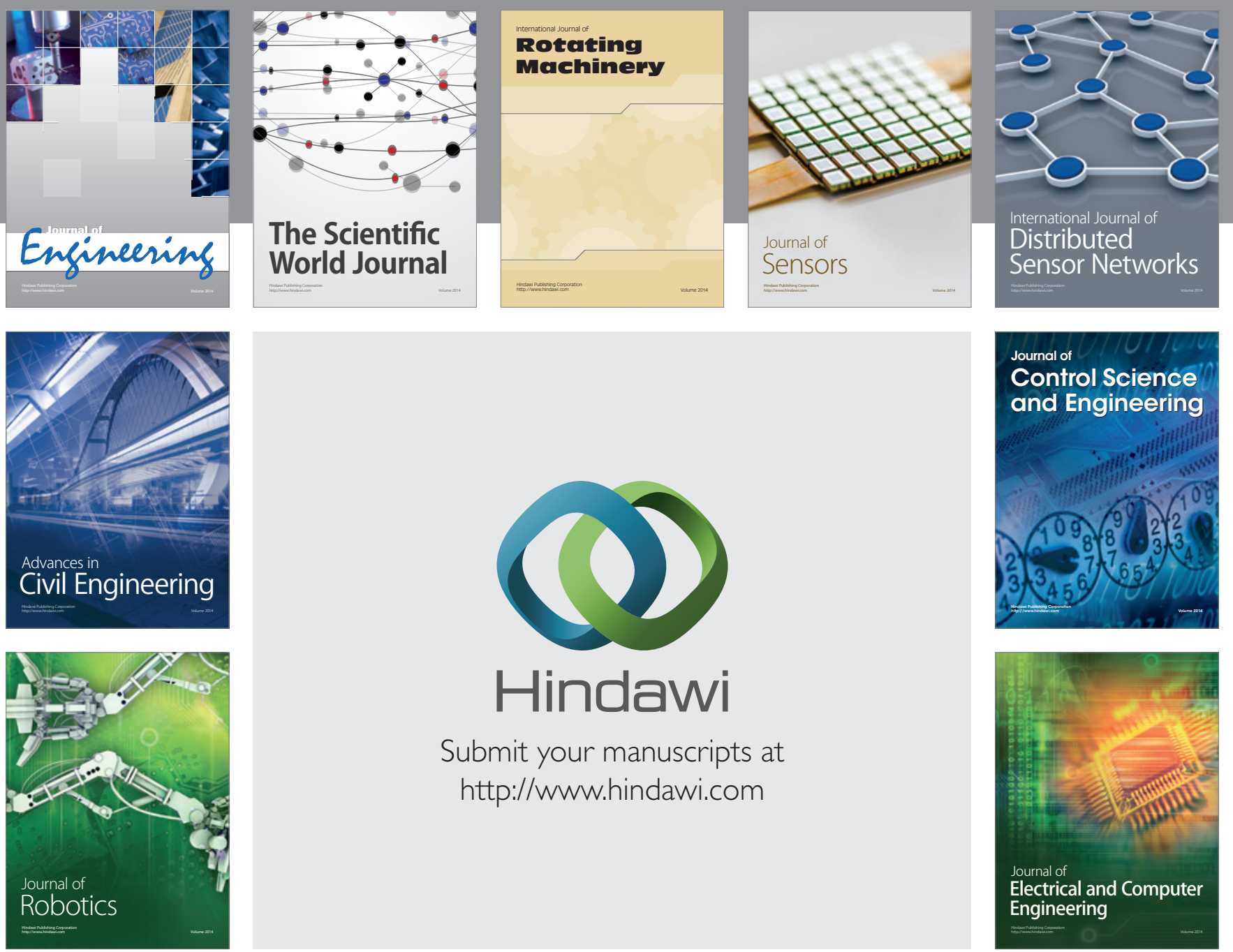

Submit your manuscripts at

http://www.hindawi.com
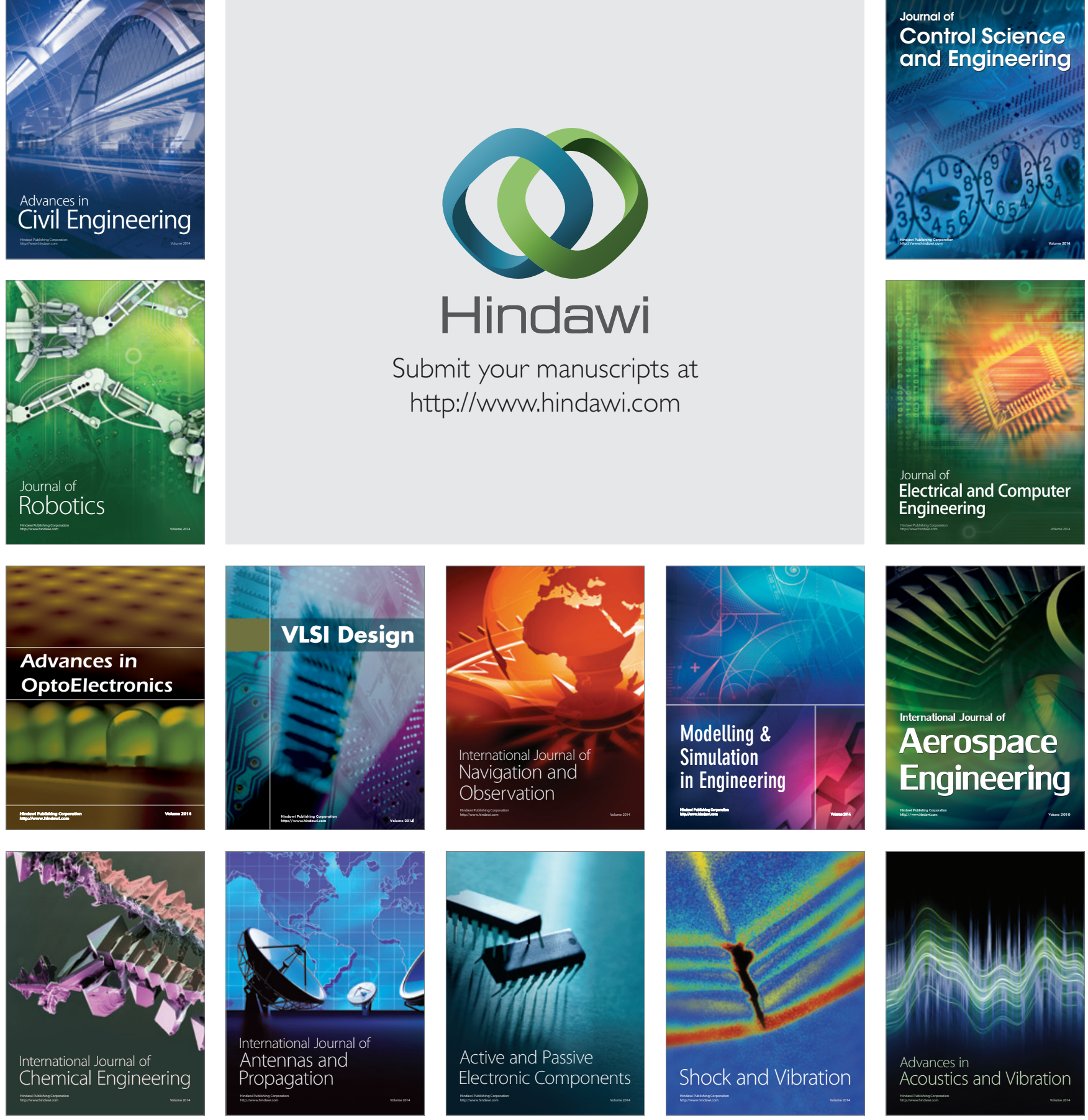